\title{
New light and shadows on Stephan's Quintet
}

\author{
C. M. Gutiérrez \\ Instituto de Astrofísica de Canarias, E-38205 La Laguna, Tenerife, Spain \\ cgc@ll.iac.es \\ M. López-Corredoira \\ Instituto de Astrofísica de Canarias, E-38205 La Laguna, Tenerife, Spain \\ Astronomisches Institut der Universität Basel, Venusstrasse 7, CH-4102 Binningen, Switzerland \\ F. Prada \\ Centro Astronómico Hispano-Alemán, Apdo. 511, E-04080 Almería, Spain \\ and \\ M. C. Eliche \\ Instituto de Astrofísica de Canarias, E-38205 La Laguna, Tenerife, Spain
}

\begin{abstract}
We present deep broad-band $R$ and narrow-band $\mathrm{H} \alpha$ images of Stephan's Quintet. The observations in the $R$ band show that the diffuse halo of the Stephan's Quintet is larger than previously thought and extends out to NGC 7320C. However, we have not found emission connecting NGC 7331 and NGC 7320 to $R \sim 26.7 \mathrm{mag} / \operatorname{arcsec}^{2}$ (at more than $3-\sigma$ level), so there is no direct evidence up to this limiting magnitude of a relation between the peculiar kinematical structure found in NGC 7331 and an ongoing or past interaction between this galaxy and NGC 7320. The $\mathrm{H} \alpha$ emission at high-velocity $\left(6000-7000 \mathrm{~km} \mathrm{~s}^{-1}\right)$ is distributed in a diffuse structure running NS between NGC 7319 and NGC 7318B and in some other more concentrated features. Some of these are located in the tidal tails produced by the interaction between the galaxies of the group. With the $\mathrm{H} \alpha$ images we have made a two-dimensional velocity map which helps to identify the origin of each structure detected. This map does not show features at intermediate velocities between the high- and low-redshift members of the group. This is in agreement with the standard scenario in which the apparent proximity of NGC 7320 to the rest of the galaxies of the Quintet is merely a projection effect. The only point which is unclear in this interpretation, is an $\mathrm{H} \alpha$ filament which is seen extending throughout NGC 7320 with velocity at $6500 \mathrm{~km} / \mathrm{s}$ instead of the $800 \mathrm{~km} / \mathrm{s}$ expected for this galaxy.
\end{abstract}

Subject headings: galaxies: individual (NGC 7317, NGC 7318A, NGC 7318B, NGC 7319, NGC 7320, NGC 7320C, NGC 7331), Galaxies: Interactions.

\section{Introduction}

Stephan's Quintet (SQ) was discovered in 1877 and is one of the most popular and studied groups of galaxies. It is classified as a compact group
(HCG 92) in the Hickson (1982) catalog. The core of the group is formed by three galaxies (NGC 7319, NGC 7318A, and NGC 7317), which have recessional velocities $\sim 6600 \mathrm{~km} \mathrm{~s}^{-1}$ with very low velocity dispersions. The other members 
of the group are NGC $7318 \mathrm{~B}$, at $\sim 5700 \mathrm{~km} \mathrm{~s}^{-1}$, and NGC 7320 at $\sim 800 \mathrm{~km} \mathrm{~s}^{-1}$. According to the orthodox view, NGC 7320 is a foreground galaxy, while NGC 7318B which has a difference in velocity of $\sim 1000 \mathrm{~km} \mathrm{~s}^{-1}$ with respect to the core of the group, shows clear signs of strong distortions which indicate ongoing interaction with the group. Two other galaxies NGC $7320 \mathrm{C}\left(\sim 6000 \mathrm{~km} \mathrm{~s}^{-1}\right)$ and NGC $7331\left(\sim 800 \mathrm{~km} \mathrm{~s}^{-1}\right)$ are not considered in the Hickson scheme as members of the group, but their apparent proximity and several reasons that we will discuss in this paper make it convenient to consider them here. A diagram showing the relative positions of the galaxies in the SQ and NGC 7331 can be found in Moles, Sulentic, \& Marquez (1997).

In spite of the large observational effort dedicated to the study of SQ from the radio to the $\mathrm{X}$ ray continuum, there are many points which yet require further attention. In particular, in this paper we address the following specific questions: $i$ ) whether the apparent proximity of NGC 7320 to the other members of $\mathrm{SQ}$ is merely a projection effect; $i i)$ whether there is any evidence of interaction between SQ (specifically NGC 7320) and NGC 7331 that could explain the particular kinematics of this galaxy (Prada et al. 1996); and iii) whether the core of SQ (NGC 7318A, NGC 7318B and NGC 7319) is connected with NGC 7320C. We have tried to answer these questions by conducting an observational program of broad- and narrow-band imaging. The narrow-band $\mathrm{H} \alpha$ images analyzed in this paper cover velocities in the range $0-8000 \mathrm{~km} \mathrm{~s}^{-1}$, while the broad-band $R$ observations correspond to two different regions: the south part of NGC7331 and SQ respectively, covering a total area $\sim 650$ square arcminutes. With the $R$ images we have investigated the possible presence of bridges between NGC 7331 and SQ, and with the narrow-band observations we have mapped the $\mathrm{H} \alpha$ and derived a map with the velocity field of this emission in SQ.

Section 2 of this paper summarizes our present knowledge and previous observations of SQ. Section 3 presents the observations and data processing. In Sections 4 and 5 we analyze the broadand narrow-band images, respectively. Section 6 discusses our results in the context of earlier observations. Our conclusions are given in Section 7.

\section{Present status of observations of Stephan's Quintet}

\subsection{Optical and near IR}

Recent studies have been conducted in the optical and near infrared from ground-based telescopes and from ISO and the Hubble Space Telescope (HST). From HST broad-band images, Gallagher et al. (2001) have recently claimed the discovery of a large number of young star clusters at distances up to $20 \mathrm{kpc}$ from the galactic center in the tidal debris of NGC 7319. This is in agreement with the standard dynamical picture (Moles, Marquez, \& Sulentic 1998), in which the ongoing interaction with NGC 7319 is older than that occurring now between NGC 7318A and NGC 7318B.

Ohyama et al. (1998) have discovered two emission-line regions in the tidal arm of NGC 7318B in a position which coincides with previously discovered radio continuum emission. The lines in these two region are at 6560 and $6720 \mathrm{~km} \mathrm{~s}^{-1}$, respectively, and much wider $\left(900 \mathrm{~km} \mathrm{~s}^{-1}\right)$ than the normal $\mathrm{H}$ II regions in NGC $7318 \mathrm{~B}$ and NGC 7319. From this, and from the the ratio between the S II and the $\mathrm{H} \alpha$ emission, they conclude that the emission is the remnant of a large number of recent supernovae. Plana et al. (1999) have presented $\mathrm{H} \alpha$ observations in SQ with a Fabry-Perot interferometer. From these, they have detected and derived the velocities for $23 \mathrm{H} \alpha$ regions with velocities in the range $5540-6700 \mathrm{~km} \mathrm{~s}^{-1}$. Mendes de Oliveira et al. (2001) and Iglesias-Páramo \& Vílchez (2001) have recently presented evidence that some of this emission corresponds to dwarf galaxies located within tidal tails in the SQ.

Yun et al. (1997) have demonstrated that SQ is deficient in molecular gas. $\mathrm{CO}$ emission is detected only in NGC 7319 and is concentrated mostly in a region $8 \mathrm{kpc}$ north of the center of the galaxy. This asymmetry could be produced by recent tidal disruptions. However Gao \& Xu (2000), using BIMA, have detected a larger abundance of CO in NGC 7319 , and also in NGC 7318B and the intergroup starburst region. Finally, Smith \& Struck (2001) have detected $\mathrm{CO}$ in a tail located to the north of $\mathrm{SQ}$ at 6000 and $6700 \mathrm{~km} \mathrm{~s}^{-1}$. This emission has $\mathrm{H} \mathrm{I}$ and $\mathrm{H} \alpha$ counterparts, and the velocities suggest that this gas corresponds to material removed from NGC 7318B and NGC 7319. 
The IRAS data (Yun et al. 1997) show that the far-infrared emission in SQ is more extended than in other groups and is remarkably similar to the X-ray emission (see below). This could indicate the presence of warm dust in the intragroupo gas.

\subsection{Radio}

Kaftan-Kassim \& Sulentic (1974) observed the radio continuum at 318,430 , and $606 \mathrm{MHz}$ and built a map of the emission which shows a connection between NGC 7331 and the source 4C33.56 across SQ. However von Kap-Herr, Haslam, \& Wielebinski (1977) observed the same region at $2695 \mathrm{MHz}$ and found no evidence of such a connection.

Allen \& Sullivan (1980) observed in the SQ region the $21 \mathrm{~cm} \mathrm{H}$ I at $750 \mathrm{~km} \mathrm{~s}^{-1}$ and $6700 \mathrm{~km} \mathrm{~s}^{-1}$ and concluded that the low redshift $\mathrm{H}$ I is entirely associated with NGC 7320, and that the highredshift $\mathrm{H}$ I is concentrated in the range 6570 $6630 \mathrm{~km} \mathrm{~s}^{-1}$. However, Sulentic \& Arp (1983) have measured the neutral hydrogen at low velocity $\left(v \leq 2200 \mathrm{~km} \mathrm{~s}^{-1}\right)$ and found that it is displaced from the optical position of NGC 7320; they argued that this has been produced by interaction between NGC 7320 and the other members of SQ. Peterson \& Shostak (1980) have observed several positions of the $21 \mathrm{~cm}$ line in the inner and outer parts of SQ and found three extended systems at velocities 5700, 6000, and $6600 \mathrm{~km} \mathrm{~s}^{-1}$. The galaxies are enveloped by a cloud of $\mathrm{H}$ I with a size of $\sim 100 \mathrm{kpc}$ (Sullivan 1980; Peterson \& Shostak 1980). Shostak, Sullivan \& Allen (1984) mapped the $\mathrm{H}$ I line in the region of SQ with a resolution of $35^{\prime \prime}$. They built a map of the gas with resolution of $50 \mathrm{~km} \mathrm{~s}^{-1}$ and confirmed the existence of three systems at velocities 5700, 6000, and $6600 \mathrm{~km} \mathrm{~s}^{-1}$. Most of the emission comes from the outer parts of the galaxies. The authors argued that the stripping of gas from the disks is due to a tidal encounter in the past and a present collision. Gillespie $(1974,1977)$ have found several radio sources $\sim 15$ arcminutes to the north of $\mathrm{SQ}$. However, the most probable interpretation is a group of radio sources at $z \sim 0.5$ without any relation with $\mathrm{SQ}$.

\subsection{X-Ray}

X-ray emission in SQ was detected (Bahcall, Harris, \& Rood 1984) by the Einstein Observatory. Other more recent observations have been obtained with ROSAT (Sulentic, Pietsch, \& Arp 1995; Pietsch et al. 1997), and $A S C A$ (Awaki et al. 1997). Pietsch et al. (1997) obtained high spatial resolution X-ray images of $\mathrm{SQ}$ with the ROSAT HRI detector. This tidal emission amounts to $\sim 5 \times 10^{41} \mathrm{erg} \mathrm{s}^{-1}$ and is distributed among the following components: $a$ ) the galaxies NGC 7319 and NGC 7318A, b) the intergalactic feature between NGC 7319 and NGC 7318B, c) a region situated $\sim 1^{\prime \prime}$ to the north of NGC 7318A,B, and $d$ ) diffuse hot gas (amounting to one third of the total emission).

\subsection{Physical picture}

The discrepant redshift of NGC 7320 has received special attention in the past. In the orthodox view, this is just a chance projection similar to those found in a large numbers of Hickson compact groups. However, some authors (Arp 1973; Burbidge \& Burbidge 1961; Sulentic \& Arp 1983) have claimed the existence of a physical connection between the low- and high-redshift galaxies of SQ. This claim is based on statistical arguments or on the numerous signs of distortions found in optical, IR and radio images of SQ. Recently, the Hubble Space Telescope has obtained images of SQ with high resolution (see http://spaceflightnow.com/news/n0010/26hststephen). It has been claimed (Moles 2001) that these images have definitely solved the question of the redshift discrepancy of NGC 7320 because it is possible to resolve individual stars in this galaxy, showing that it is closer than the rest of the galaxies of the group. However, Arp (2001) (private comm.), in strong disagreement with this, claims that in these images it is also possible to resolve individual stars in the high-redshift members of the group. Furthermore, using the luminosity of the H II region as a distance indicator, Arp (2001) (private comm.) points out that the low- and high-redshift galaxies of SQ should be at the same distance.

The other relevant fact in the discussion on SQ is the large difference in velocity $(\sim 1000 \mathrm{~km}$ $\mathrm{s}^{-1}$ ) between NGC $7318 \mathrm{~B}$ and the rest of the high- $z$ members of SQ. Moles et al. (1998) argue 
that NGC $7318 \mathrm{~B}$ is crossing the group now; as this galaxy has kept its gas, this current crossing should be occurring for the first time. As a consequence of this encounter, the interstellar medium of NGC 7318B is shocking the intragroup medium, especially in the Northern Starburst Forming (NSF) region (Gallagher et al. 2001) and in the tidal tail of NGC 7319. As is expected from numerical simulations and as an increasing number of observations show, this interaction is producing intense starbursts in several regions of the group. There are several features indicating that this interaction is in fact in progress; for instance: $i$ ) optically, NGC 7318A and NGC 7318B look like a typical pair merging, $i i$ ) there is radio emission associated with the interaction between NGC 7319 and NGC 7318B, and iii) a large number of knots has been observed in the arms of NGC 7318B. However, Arp (1973) has suggested that with this difference in velocities the group would dissipate over a very short timescale so that it would be very unlikely to see them together.

In the standard picture (Moles et al. 1998), NGC $7320 \mathrm{C}$ crossed the group $10^{8}$ years ago producing considerable disruptions; for instance, it removed the $\mathrm{H}$ I in NGC 7319 creating a visible tail seen today in this galaxy, with part of the gas being deposited in a region northeast of NGC 7319. The interaction was also probably responsible for the Seyfert 2-type nuclear activity of NGC 7319. In this paper, we will show more evidence of the connection between NGC 7320C and the other galaxies in SQ.

\section{Observations and data reduction}

The observations analyzed in this paper were obtained during several runs between 1997 and 2000 on the $0.8 \mathrm{~m}$ IAC 80 telescope at Teide Observatory $^{1}$, the $2.2 \mathrm{~m}$ telescope at Calar Alto $^{2}$, and the $2.5 \mathrm{~m}$ Isaac Newton Telescope (INT) at Roque de los Muchachos Observatory on La Palma (Spain) ${ }^{3}$. The observations taken with the IAC 80

\footnotetext{
${ }^{1}$ The IAC 80 is operated by the Instituto de Astrofísica de Canarias at Teide Observatory on the island of Tenerife (Spain).

${ }^{2}$ The German-Spanish Astronomical Centre, Calar Alto, is operated jointly by the Max-Planck-Institute for Astronomy, Heidelberg, and Spain's National Commission for Astronomy.

${ }^{3}$ The INT is operated by the Isaac Newton Group.
}

Table 1: Summary of the observations

\begin{tabular}{cccc}
$\lambda_{\text {cent }}(\AA)$ & FWHM $(\AA)$ & Tel. & $T_{\exp }(\mathrm{s})$ \\
\hline 6611 & 50 & IAC 80 & 11700 \\
6687 & 50 & IAC 80 & 5400 \\
6724 & 50 & IAC 80 & 4800 \\
6569 & 113 & Calar $2.2 \mathrm{~m}$ & 1000 \\
6667 & 76 & Calar $2.2 \mathrm{~m}$ & 1000 \\
6737 & 66 & Calar $2.2 \mathrm{~m}$ & 1000 \\
$R$ & & INT & 2200 \\
$R$ & & IAC 80 & 4800
\end{tabular}

and the $2.2 \mathrm{~m}$ telescope, we concentrated specifically on the $\mathrm{H} \alpha$ emission in $\mathrm{SQ}$ (the $R$ images taken with the IAC 80 were used only for continuum subtraction of the $\mathrm{H} \alpha$ images). The south of NGC 7331 and SQ were observed in the $R$-band with the wider field of the WFC on the INT, with a total exposure time $\sim 2000$ s each. Typical seeing was $\sim 2$ arcsec in the narrow band images, and $\sim 1$ arcsec for the $R$-band observations. Table 1 summarizes the observations. The spectral response of the narrow-band filters can be found at http://www.iac.es/telescopes/ten.html and http://www.caha.es/CAHA/Instruments/filterlist.html. We performed standard data reduction: bias subtraction, flat-field correction, coaddition of images for the same filter, and sky subtraction. Only the observations at the INT have been photometrically calibrated, using stars from the catalog of Landolt et al. (1992). For the observations in the $\mathrm{H} \alpha$ narrow bands we subtracted the continuum using the images in $R$ taken with the IAC 80 or in a different redshifted $\mathrm{H} \alpha$, scaling according to the flux measured in field stars.

\section{Broad-band imaging}

Images in the $R$ band were taken with the Wide Field Camera at the $2.5 \mathrm{~m}$ INT at Roque de los Muchachos Observatory in 1997 August. The observations covered $\sim 1800$ square arcminutes from which we have selected $\sim 650$ square arcminutes corresponding to the SQ region and the southern part of the galaxy NGC 7331. We had two 
objectives: the first was to delineate the halo of $\mathrm{SQ}$, and the second was to search for possible evidence of interaction between NGC 7331 and the other SQ galaxies. The limiting $3 \sigma$ magnitude per 0.37 square arcsecond pixels was $\sim 25.2$ $\mathrm{mag} / \operatorname{arcsec}^{2}$. However, the structures that we wanted to map were diffuse and spatially very extended, so we binned the images to a resolution $\sim 2.5$ arcsecs, this permits an enhancement of the signal-to-noise ratio by a factor $\sim 7$, that is, we go almost two magnitudes deeper, being now the 3- $\sigma$ limiting magnitude $27.3 \mathrm{mag} / \mathrm{arcsec}^{2}$. Figures $1 a, b$ present the gray-scale and isophotal map obtained around SQ and in the southern part of NGC 7331 respectively. Figure $1 a$ shows that the halo is considerable more extended that it was revealed in the observations by Moles et al. (1998). For the first time, we have found evidence of a clear connection in the form of diffuse light between SQ and the galaxy NGC 7320C. In particular the SW tail which emerges from SQ seems to extend to this galaxy. We think that this supports the previously suspected connection between SQ and NGC 7320C. We have traced the emission up to the isophote at $26.7 \mathrm{mag} / \operatorname{arcsec}^{2}$ and 25.5 $\mathrm{mag} / \operatorname{arcsec}^{2}$ around the SQ and NGC 7331 respectively. The $25.5 \mathrm{mag} / \operatorname{arcsec}^{2}$ isophote surrounds and seems to enclose NGC 7331. These numbers are conservative with respect to the sensitivity of the image and take into account the uncertainties in the estimation of the sky level, particularly difficult in the observations of the region around NGC 7331.

There are no signs of past or present interaction in the form of tails, bridges, etc., between SQ and NGC 7331. We would remark that the last isophote surrounding SQ is not associated with any individual galaxy, but instead corresponds to the diffuse halo of the group, while the last isophote detected around NGC 7331 is more regular, roughly following the shape of the galaxy, and seems to be associated with the disk (and halo) of this galaxy.

\section{H $\alpha$ maps and velocity field}

To differentiate the emission coming from the low-z (at the systemic velocity of NGC 7320) and high-z (the other galaxies of SQ) respectively, we decided to take $\mathrm{H} \alpha$ images redshifted at the veloc- ities of both components (see Section 3). Figure 2 presents the $R$ band (gray-scale) and the total $\mathrm{H} \alpha$ (contours). The $\mathrm{H} \alpha$ maps presented here are a combination of the emission associated with the low- and high- $z$ components. To enhance the signal with respect to the noise we binned the data to a common pixel size of 1.06 arcsec; this produces a slight degradation in resolution. There are many spurious $\mathrm{H} \alpha$ features associated with residuals due to imperfect continuum subtraction in the stars of the field. In general, there is no ambiguity in distinguishing between these residuals and real $\mathrm{H} \alpha$ emission because the latter is specifically associated with several extended features that we discuss below. Figure 3 presents the images in the narrow filters observed combining the $\mathrm{H} \alpha$ low- $z$ (contours) and high- $z$ (gray-scale) filters, respectively. The size of the images is $\sim 6 \times 6 \operatorname{arcmin}^{2}$. From these images, it is clear that the low- $z$ emission is associated with NGC 7320. The high- $z$ emission is concentrated in three well differentiated structures: $i$ ) the center of NGC 7319, ii) an intergroup feature in the NS direction between NGC 7318B and NGC 7319, and iii) a structure to the north of the aforementioned feature, which corresponds to features previously identified by Xu, Sulentic, \& Tuffs (1999) and Gallagher et al. (2001). Apart from these components, there are several other high- $z \mathrm{H} \alpha$ features in the tidal tails produced by the strong interaction within the group. From Fig. 3, we see that the lowand high- $z$ emission are associated with different spatial regions, and that there are no regions in which both types of emission overlap. Although our spatial resolution is poor and the $\mathrm{H} \alpha$ emission is diffuse, using the algorithm SExtractor (Bertin \& Arnouts 1996) and eliminating the spurious detections associated with star residuals, we identified about 20 and 30 separated regions in the lowand high- $z$ maps, respectively. The low- $z$ structures are symmetrically distributed in the disk of NGC 7320 (see however Section 6.1) and tend to be isolated and compact. The high- $z$ emission tends to be more diffuse than the low- $z$ component. This is in agreement with the standard picture in which components $i i$ and $i i i$ (see above) of the high- $z$ emission are associated with the intergroup medium formed by material stripped by recent and ongoing strong interaction involving the galaxies NGC 7318A, NGC 7318B, NGC 7319, and 
NGC 7320C.

We may consider the set of $\mathrm{H} \alpha$ images as a two-dimensional spectrum with very low resolution. It then makes sense to compute the centroid and dispersion of a given feature. From this, it is straightforward to produce a two-dimensional velocity map. We proceed as follows. A single wavelength, $\lambda_{i}$, was assigned to the $i$ th pixel of the image:

$$
\lambda_{i}=\frac{\sum_{j} F_{i j} \times \lambda_{j}}{\sum_{j} F_{i j}}
$$

where $F_{i j}$ the flux in pixel $i$ and filter $j$, and $\lambda_{j}$ is the central wavelength in pixel $i$. To eliminate most of the residuals due to stars, only fluxes at a level above $2 \sigma$ were considered. From this, we computed the associated velocity as $v_{i}=$ $\left(\lambda_{i}-\lambda_{0}\right) / \lambda_{0}\left(\lambda_{0}=6562 \AA\right)$. Figures $4 a$ and $b$ show the velocity and the associated error map, respectively. Overplotted in Fig. $4 a$ is the ellipse which fits the outer isophote of NGC 7320 in the $R$-band. An important result is that the velocities derived are in two well separated ranges: one from 5500 to $7500 \mathrm{~km} \mathrm{~s}^{-1}$, and the other from 700 to $1000 \mathrm{~km}$ $\mathrm{s}^{-1}$. No features at intermediate velocities that could suggest a Doppler redshift as the origin of the difference in velocities between NGC 7320 and the rest of the SQ have been detected. However, some points concerning the relationship between these two features are unexplained and are discussed in next section.

\section{Discussion}

The relation between NGC 7331 and NGC 7320 is clear according to their relative positions and systemic velocities. From the $\mathrm{H}$ I line the difference in velocities between both galaxies is $\sim 45 \mathrm{~km}$ $\mathrm{s}^{-1}$ (de Vaucouleurs et al. 1991). Their projected separation is $\sim 100 \mathrm{kpc}$, and the magnitudes in the $B$ band are 9.38 and 13.56 for NGC 7331 and NGC 7320, respectively. These allow us to classify NGC 7320 as a satellite of NGC 7331 according to the criteria defined by Zaritsky et al. (1997). We have searched the NED for objects at less than 500 $\mathrm{kpc}$ from NGC 7331 and with recessional velocities similar to that of this galaxy and found another two galaxies besides NGC 7320. These galaxies seem to constitute one of the loose groups identified by Trasarti-Battistoni (1998). At angular dis- tances $\leq 1$ degree $(\leq 2 \mathrm{Mpc})$ from $\mathrm{SQ}$, there are about ten other galaxies with measured redshifts and with recessional velocities in the range 5500$7500 \mathrm{~km} \mathrm{~s}^{-1}$; they form another loose group with 9 members identified by Trasarti-Battistoni (1998); a subgroup of such galaxies (NGC7335, NGC7337, and NGC7340) at the East of NGC7331 has been classified as a poor cluster by White et al. (1999).

One of the mechanisms proposed to explain the counter-rotating core found in NGC 7331 (Prada et al. 1996) is the interaction of this galaxy with a small satellite. Although this could really be the origin of such kinematics, in our deep $R$ images we have found no evidence (bridges, tails, etc.) to $R \sim 26.7 \mathrm{mag} / \operatorname{arcsec}^{2}$ supporting such an interaction between NGC 7331 and NGC 7320 .

We have carried out a comparative study between the features detected in our velocity map and the results by Plana et al. (1999). The position of these common $\mathrm{H} \alpha$ regions is indicated in Fig. 3. We have indicated also in the figure two features in one of the tails connecting NGC 7320c with the rest of SQ. The comparison with the results by Plana et al. (1999) shows that we have detected and estimated the velocities of the regions detected by these authors (the detection of region number 23 it is not completely clear in our data), although we have found a systematic difference of $\sim+300 \mathrm{~km} \mathrm{~s}^{-1}$ in the estimated velocities. This is consistent with the uncertainties expected from the typical width of the $\mathrm{H} \alpha$ filters used in our analysis. In Table 2 we present our estimate of the velocities of $\mathrm{H} \alpha$ regions detected by Plana et al. (1999) (we have adopted the notation used by these authors). This uncertainty is too large to distinguish between the components associated with NGC 7319 and the other three galaxies (NGC 7318A, NGC 7318B, and NGC 7317), but is small enough to distinguish between emission associated with the above four galaxies and NGC 7320. Due to the technique used, in some cases Plana et al. (1999) were not able to measured unambiguously the velocities, so our observations represent the first determination of such velocities. Our analysis allows us to distinguish only one component in velocity in each position. If there were more than one, this should reveal itself in our velocity map as an increase in the pixel-topixel rms according to the importance of one or other component. This seems to be the case for 
region 13 (in the notation of Plana et al. 1999) in which these authors detected two different components, and which shows up in our map as a large variation in velocity from pixel to pixel.

Some of these features are in the tidal tails resulting from the strong interaction within the group. This is in perfect agreement with the results of Iglesias-Páramo \& Vílchez (2001), who identified these features as dwarf galaxies. The above results are also in agreement with the predictions from numerical simulations (Barnes 1988) in which dwarf galaxies can form in tidal tails resulting from the interaction of two disk galaxies.

Our broad-band observations reveal a bigger halo that was previously observed. As pointed out by Moles et al. (1998), this light is not associated with the individual galaxies, but is instead diffuse light associated with present and past interactions within the group. In particular it indicates large transversal motions of NGC 7317 and NGC 7320C. The interaction with this galaxy seems to be responsible for the diffuse light observed to the north of SQ. The extension of the SW tail to NGC 7320C strongly supports the standard scenario proposed by Moles et al. (1998), in which the galaxy crossed SQ and created this tail, which runs in parallel to a second tail which emerges from one of the arms of NGC 7319. This second tail is brighter as it would be expected if it corresponds to a past encounter with the core from which most of the gas associated with NGC 7319 (and partially with NGC 7320C) was stripped.

Table 2: Recessional velocities (in $\mathrm{km} \mathrm{s}^{-1}$ ) of the $\mathrm{H} \alpha$ regions in common with Plana et al. (1999).

\begin{tabular}{ccccccc}
\hline Region & 1 & 2 & 3 & 4 & 5 & 6 \\
Velocity & 6300 & 6000 & 6300 & 6300 & 6300 & 7200 \\
\hline Region & 7 & 8 & 9 & 10 & 11 & 12 \\
Velocity & 6900 & 6400 & 6900 & 6300 & 6250 & 6400 \\
\hline Region & 13 & 14 & 15 & 16 & 17 & 18 \\
Velocity & 6100 & 6100 & 5500 & 5950 & 5750 & 6100 \\
\hline Region & 19 & 20 & 21 & 22 & 23 & \\
Velocity & 6000 & 6200 & 5800 & 5750 & $6100 ?$ &
\end{tabular}

\subsection{A remark on some odd coincidences}

Apart from all the positive facts which suggest that NGC 7320 is unrelated to the rest of the components of SQ, as we have argued above, there is one further observational aspect whose interpretation is not clear in the light of the orthodox view: there is an $\mathrm{H} \alpha$ bridge (see Figs. 3, $4 a$ and $4 c$ ) which is a continuation of the intergalactic gas of the high-redshift galaxies of SQ pointing to NGC 7320 and extending throughout this galaxy. In particular, we would like to remark:

- The emission of $\mathrm{H} \alpha$ at $6500 \mathrm{~km} / \mathrm{s}$ is in a region of NGC7320 where there is no emission of $\mathrm{H} \alpha$ at $800 \mathrm{~km} / \mathrm{s}$. Assuming elliptical symmetry for NGC 7320 (such symmetry exists in the $R$-band), we would expect to observe $\mathrm{H} \alpha$ emission with both velocities $(6500 \mathrm{~km}$ $\mathrm{s}^{-1}$ and $800 \mathrm{~km} \mathrm{~s}^{-1}$ ) superposed in the region of the bridge. Instead of that, we observe a gap in the $\mathrm{H} \alpha$ emission at $800 \mathrm{~km} / \mathrm{s}$ precisely where the bridge at $6500 \mathrm{~km} / \mathrm{s}$ is present.

- Exactly in the point where the bridge of 6500 $\mathrm{km} / \mathrm{s}$ finishes, HII regions at $800 \mathrm{~km} / \mathrm{s}$ begin. We might think that the opacity of these HII regions does not allow to see what is behind. However, it is not only in the position of the HII regions at $800 \mathrm{~km} / \mathrm{s}$, but also in the gaps between them, where we do not find any emission at $6500 \mathrm{~km} / \mathrm{s}$.

These coincidences could be explained naturally if a bridge at $6500 \mathrm{~km} / \mathrm{s}$ were in front of the galaxy at $800 \mathrm{~km} / \mathrm{s}$. Although the evidence is not conclusive, and difficult to fit in an ortodox view, we think that this is a new and interesting observational fact to consider in future studies of SQ.

\section{Conclusions}

1) We have obtained deep $R$ images in the region from SQ to the southern edge of the giant spiral NGC 7331. We have found no evidence of an optical connection in the form of tails, bridges, etc., between SQ and NGC 7331 above magnitudes $\sim 26.7 \mathrm{mag} / \operatorname{arcssec}^{2}$.

2) We have delineated the halo of SQ up to the above magnitude showing that it extends to and 
contains the galaxy NGC 7320C. Additionally, in the $R$-band image, a tail connecting the core of SQ and NGC $7320 \mathrm{C}$ is seen. This is interpreted as a tidal tail produced by a past encounter between NGC 7320C and the core of the group.

3) With narrow-band images in the range 0$8000 \mathrm{~km} \mathrm{~s}^{-1}$, we have delineated the $\mathrm{H} \alpha$ emission. We see that this is concentrated in a feature in the NS direction situated between NGC 7318B and NGC 7319 , a region $\sim 1$ arcminute to the north of NGC 7318B and the nucleus of NGC 7319.

4) With the $\mathrm{H} \alpha$ images, we have obtained a low-resolution velocity map of the SQ region. The $\mathrm{H} \alpha$ images and the velocity map show evidence of emission in the tidal tail that is the result of the strong interaction within the group.

5) The velocity map derived from the $\mathrm{H} \alpha$ images does not show features at intermediate velocities between the low- and the high- $z$ components.

6) A filament in $\mathrm{H} \alpha$ at $\sim 6500 \mathrm{~km} \mathrm{~s}^{-1}$ apparently connecting NGC 7320 (at $\sim 800 \mathrm{~km} \mathrm{~s}^{-1}$ ) and the rest of SQ is seen. We think that this fact leaves the point of the possible relation between NGC 7320 and the rest of SQ still open and will require further attention.

We wish to thank J. Iglesias-Páramo, who kindly provided the $\mathrm{H} \alpha$ images taken at the $2.2 \mathrm{~m}$ telescope in Calar Alto.

\section{REFERENCES}

Allen, R. J., \& Sullivan, W. T., III 1980, A\&A, 84,181

Arp, H. 1973, ApJ, 183, 411

Awaki, H., Koyama, K., Matsumoto, H., Tomida, H., Tsuru, T., \& Ueno, S. 1997, PASP, 49, 445

Bahcall, N. A., Harris, D. E., \& Rood, H. J. 1984, ApJ, 284, L29

Barnes, J. E. 1988, ApJ, 331, 699

Bertin, E., \& Arnouts, S. 1996, AAS, 117, 393

Burbidge, E. M., \& Burbidge, G. R. 1961, ApJ, 134,244

de Vaucouleurs, G., de Vaucouleurs, A., Corwin, JR, H. G., Buta, R. J., Paturel, G., \& Fouque,
P. 1991, Third Reference Catalogue of Bright Galaxies. Springer-Verlag. N.Y.

Gallagher, S. C., Charlton, J. C., Hunsberger, S. D., Zaritsky, D., \& Whitmore, B. C. et al. 2001, AJ, 122, 163

Gao, Y. \& Xu, C. 2000, ApJ, 542, L83

Gillespie, A. R. 1974, MNRAS, 166, 11

Gillespie, A. R. 1977, MNRAS, 181, 149

Hickson, P. 1982, ApJ, 255, 382

Iglesias-Páramo, J., \& Vílchez, J. M. 2001, ApJ, 550, 204

Kaftan-Kassim, M. A. \& Sulentic, J. W. 1974, A\&A, 33, 343

Landolt, A. U. 1992, A\&A, 104, 340

Mendes de Oliveira, C., Plana, H., Amram, P., Balkowski, C., \& Bolte, M. 2001, AJ, 121, 2524

Moles, M. 2001 http://spaceflightnow.com/news/ n0010/26hststephen

Moles, M. Marquez, I., \& Sulentic, J. W. 1998, A\&A, 334, 473

Moles, M., Sulentic, J. W., \& Marquez, I. 1997, ApJ, 485, L69

Ohyama, Y., Nishiura, S., Murayama, T., \& Taniguchi, Y. 1998, ApJ, 492, L25

Peterson, S. D. \& Shostak, G. S. 1980, ApJ, 241, L1

Pietsch, W., Trinchieri, G., Arp, H., \& Sulentic, J. W. 1997, A\&A, 322, 89

Plana, H., Mendes de Oliveira, C., Amram, P., Bolte, M., Balkowski, C., \& Boulesteix, J. 1999, ApJ, 516, L69

Prada, F., Gutiérrez, C. M., Peletier, R. F., \& McKeith, C. D. 1996, ApJ, 463, L9

Shostak, G. S., Sullivan, W. T. III, \& Allen, R. J. 1984, A\&A, 139, 15

Smith, B. J. \& Struck, C. 2001, AJ, 121, 710

Sulentic, J. W., \& Arp. H. 1983, AJ, 88, 267 
Sulentic, J. W., Pietsch, W., \& Arp, H. 1995, ApJ, 445,85

Sullivan, W. T. III 1980, A\&A, 89, L3

Trasarti-Battistoni R. 1998, AAS, 130, 341

von Kap-Herr, A., Haslam, C. G. T., \& Wielebinski, R. 1977, A\&A, 57, 337

White, R. A., Bliton, M., Bhavsar, S. P., Bornmann, P., Burns, J. O., Ledlow, M. J., \& Loken, C. 1999, AJ, 118, 2014

Xu, C., Sulentic, J. W., \& Tuffs, R. 1999, ApJ, 512,178

Yun, M. S., \& Verdes-Montenegro, L., del Olmo, A., \& Perea, J. 1997, ApJ, 475, L21

Zaritsky, D., Smith, R., Frenk, C., \& White, S. D. M. 1997, ApJ, 478, 39

This 2-column preprint was prepared with the AAS IATEX macros v5.0. 


\section{Figures}

Fig. 1.- Gray-level and contour $R$ map in the region of Stephan's Quintet and in the southern part of NGC 7331. The lowest isophotes are 26.7 and $25.5 \mathrm{mag} / \operatorname{arcsec}^{2}$, respectively.

Fig. 2.- Broad-band $R$ filter (gray-scale) and $\mathrm{H} \alpha$ emission (contours) of Stephan's Quintet.

Fig. 3.- Low- $z$ (contours) and high- $z$ (gray-scale) $\mathrm{H} \alpha$ maps in the region of Stephan's Quintet.

Fig. 4.- (a) Velocity and (b) error maps in Stephan's Quintet region obtained from the low- $z$ and high- $z \mathrm{H} \alpha$ images. (c) An expanded view of the velocity map centered on the region of abrupt transition from the low to the high- $z$ emission. 
This figure "f1a.jpg" is available in "jpg" format from: http://arxiv.org/ps/astro-ph/0202521v1 
This figure "f1b.jpg" is available in "jpg" format from: http://arxiv.org/ps/astro-ph/0202521v1 
This figure "f2.jpg" is available in "jpg" format from: http://arxiv.org/ps/astro-ph/0202521v1 
This figure "f3.jpg" is available in "jpg" format from: http://arxiv.org/ps/astro-ph/0202521v1 
This figure "f4a.jpg" is available in "jpg" format from: http://arxiv.org/ps/astro-ph/0202521v1 
This figure "f4b.jpg" is available in "jpg" format from: http://arxiv.org/ps/astro-ph/0202521v1 
This figure "f4c.jpg" is available in "jpg" format from: http://arxiv.org/ps/astro-ph/0202521v1 